\title{
Estimation Formulas for Embedded-length of Connection Embedded-type Foundation Unit Modular System
}

\author{
Keum-Sung Park ${ }^{1}$, Sang-Sup Lee ${ }^{2}$, Sung-Yub Hong ${ }^{3}, \quad$ Kyu-Woong Bae ${ }^{4}$ \\ ${ }^{1}$ Research Fellow at the Korea Institute of Civil Engineering and Building Technology \\ ${ }^{2}$ Senior Research at the Korea Institute of Civil Engineering and Building Technology \\ ${ }^{3}$ Post-Master Research at the Korea Institute of Civil Engineering and Building Technology \\ ${ }^{4}$ Senior Research Fellow at the Korea Institute of Civil Engineering and Building Technology \\ *Corresponding author's e-mail: kspark1@kict.re.kr
}

\begin{abstract}
Modular unit structure system has been used increasingly for mid-rise buildings where repetitive units are required. By assembling the pre-made modular unit structures, a building can be easily constructed. The assembled building must behave as one single structure to effectively transfer the forces and moments developed by gravity and lateral loads. For this, individual modular unit structure has to be properly connected to each other. Therefore, evaluation of the connection behavior of modular unit structure system is crucial. This study proposed the embedded steel column-to foundation connection for modular unit structure system and evaluated the behavior of the proposed connection. A series of experiments was performed to investigate the effect of embedment depth of the column, the shape of the end plate, and the shear studs in the embedded region of the column on the connection behavior. Finally, a design equation for the proposed connection was suggested.
\end{abstract}

\section{KEYWORDS}

Unit Modular, Foundation, Embedded Column, Flexural Performance, Connection Design

\section{Introduction}

This study presents new type of unit modular systems and column-foundation connection detail which is developed in Korea Institute of Civil Engineering and Building Technology (KICT). Then, the failure mechanism of the proposed connection was investigated through a series of test and proposed the design equations.

Fig. 1 shows a unit modular system and a building structure constructed by the unit modular system. As shown in this figure, a structure can be easily built by assembling the unit modular system, which is manufactured in a factory. Thus kind of unit modular systems have been widely applied to middle-rise buildings which require same units repeatedly such as hospitals, schools, dormitories, hotels, or worker's accommodation. The building made by unit modular structure is popular in the Northern Europe region (Annan CD, 2009). Recently, studies about unit modular systems to apply to high-rise buildings have been conducted (Lawson RM, 2012).

The following advantages can be obtained using the unit modular systems. (a) Economical building construction can be facilitated using a simple and repeated structure. (b) Improvements on constructability and shortening of construction scheduling can be achieved. (c) Quality improvement can be achieved through factory manufacturing of unit modular systems (Lawson RM, 2010). 

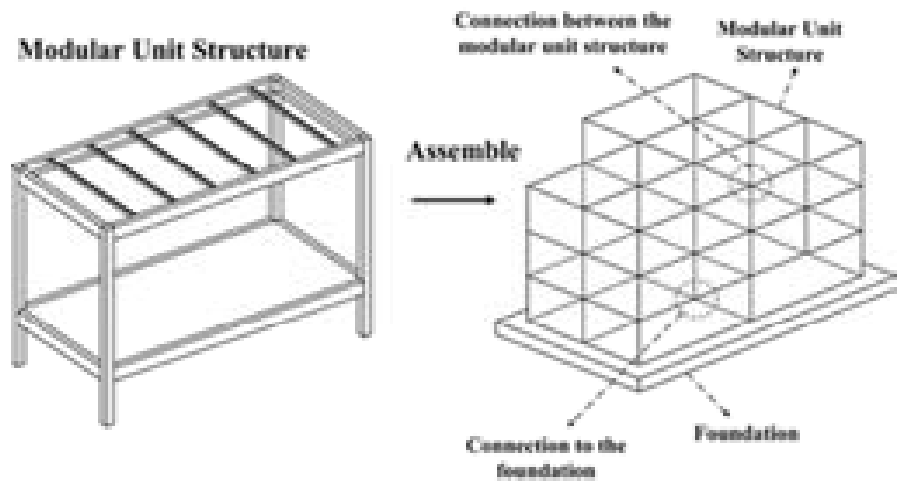

Figure 1 Unit modular system and building assembled using unit modular structures.

The connection of unit modular system can be divided into three parts as shown in Fig. 1. The first one is a connection between column and beam, the second is a connection between each unit modular structure, and the third is a connection between unit modular system and foundation. The connection must transfer the forces and moments appropriately so that the assembled building acts as one single structure. However, the studies about the connection of unit modular system are limited in the literatures.

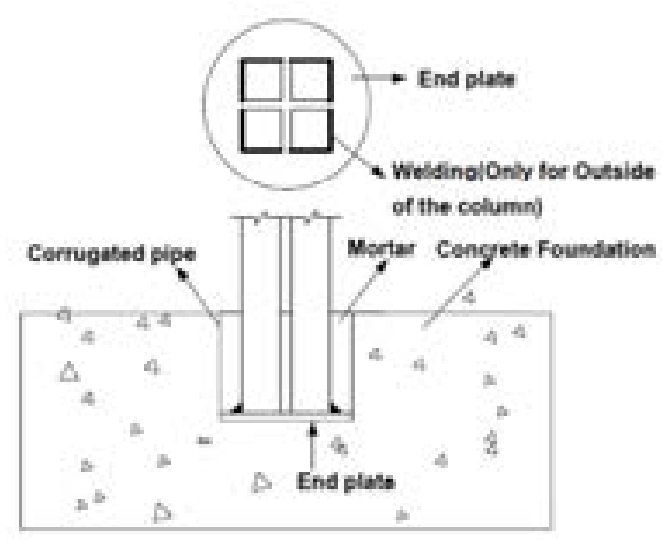

Figure 2 proposed embedded connection for column of unit modular structure to the foundation.

The connection between column of unit modular structure and the foundation should provide plastic bending moment strength of the column and sufficient ductility to resist the unexpected lateral forces, such as earthquake loading. For this, embedded connections shown in Fig. 2 have been proposed based on the embedded CFT column to foundation connections proposed by Lehman et al. (2012) and Moon et al. (2013).

As shown in Fig. 2, outer perimeters of columns are welded to the base plate. Then, the base plate is embedded into the footing. Finally, void part is grouted. It should be noted that the columns are not mechanically connected and there is gap between the columns.

In this study, a series of experimental studies was conducted to investigate the failure mechanism of the proposed connection. The major parameter is embedment depth of the column. Finally, simple design equation for the proposed connection was suggested. 


\section{Experiment}

\subsection{Overview of the experiment specimen}

Table 1 shows the dimensions of the specimen used in this study. Five specimens were constructed. The main parameter is the embedment depth. The depth varies from $125 \mathrm{~mm}$ to $225 \mathrm{~mm}$. In Table $1, b_{1}$ is the width of the column, where $b_{1}$ is $125 \mathrm{~mm}$ for all specimens. The column has a rectangular cross-section and the thickness $(t)$ is $6 \mathrm{~mm}$. As shown in Figure 3, four modular columns were welded to the base plates.

The base plates of specimens MFC125-c, MFC175-c, MFC225-c, and MFC175-cs are circular shape while that of MFC175-d is diamond shape. A thickness of the base plates $\left(t_{b}\right)$ is $6 \mathrm{~mm}$ for all specimens. For MFC175-cs, a shear stud was installed in the embedded foundation to investigate the effect of the shear stud on the connection behavior.

Table 1 Dimensions of the test specimens

\begin{tabular}{|c|c|c|c|c|c|c|c|}
\hline \multirow[b]{2}{*}{ Name } & \multirow[b]{2}{*}{$\begin{array}{c}b_{1} \\
(\mathrm{~mm})\end{array}$} & \multirow[b]{2}{*}{$\begin{array}{c}t \\
(\mathrm{~mm})\end{array}$} & \multirow[b]{2}{*}{$\begin{array}{c}l_{e} \\
(\mathrm{~mm})\end{array}$} & \multirow[b]{2}{*}{$l_{e} / 2 b_{1}$} & \multicolumn{2}{|c|}{ base plate } & \multirow[b]{2}{*}{ Stud } \\
\hline & & & & & $\begin{array}{c}t_{b} \\
(\mathrm{~mm})\end{array}$ & Shape & \\
\hline MFC125-c & 125 & 6.0 & 125 & 0.50 & 6.0 & Circular & $X$ \\
\hline MFC175-c & 125 & 6.0 & 175 & 0.70 & 6.0 & Circular & $X$ \\
\hline MFC225-c & 125 & 6.0 & 225 & 0.90 & 6.0 & Circular & $X$ \\
\hline MFC175-d & 125 & 6.0 & 175 & 0.70 & 6.0 & Diamond & $X$ \\
\hline MFC175-cs & 125 & 6.0 & 175 & 0.70 & 6.0 & Circular & $\mathrm{O}$ \\
\hline
\end{tabular}

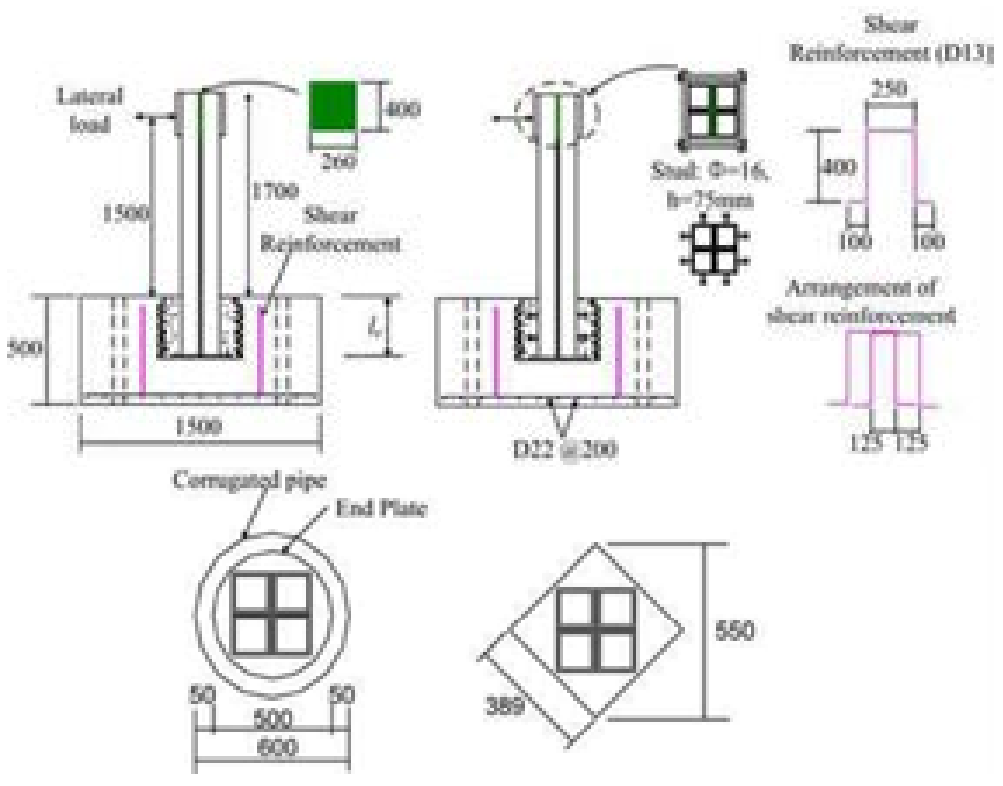

Figure 3 Details of test specimens. 
Fig. 3 shows the detailed shape and dimension of the specimen. The width of the foundation was 1,500 $\mathrm{mm}$ and the depth of the foundation was $500 \mathrm{~mm}$. The diameter of the circular base plate was $500 \mathrm{~mm}$. In the foundation, flexural and shear reinforcement was placed as shown in Fig. 3. For flexural reinforcement, D22 reinforcement was placed with $200 \mathrm{~mm}$ spacing, while D13 reinforcement was used for shear reinforcement. The nominal diameter for D22 and D13 reinforcement are $22 \mathrm{~mm}$ and $13 \mathrm{~mm}$, respectively.

Only lateral force was applied at the top of the specimens, since flexural strength of steel column is largest when a compressive force is zero. The compressive strength of the concrete in the foundation was $27 \mathrm{MPa}$. The yield and ultimate stress of the steel were $235 \mathrm{MPa}$ and $400 \mathrm{MPa}$, respectively.

\subsection{Experiment method}

Fig. 4 shows the test setup and loading protocol. Test specimen was fixed with four anchors and the cyclic lateral loading was applied at the top of the specimen. The loading protocol used in this study selected based on the AISC “Seismic Provisions for Structural Steel Building” provisions (AISC, 2005).
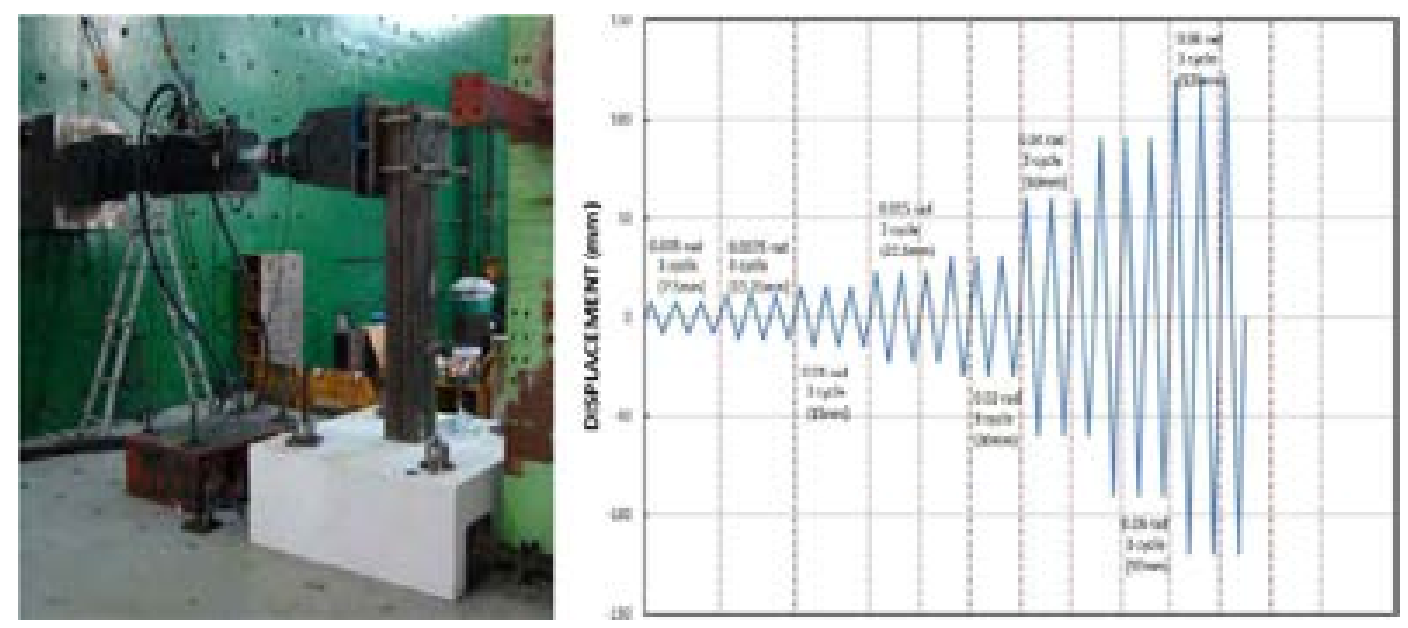

Figure 4 test setup and loading protocol.

\subsection{Experiment result analysis}

\subsubsection{Effect of the embedded depth}

Fig. 5 shows a moment-drift ratio relationship of MFC125-c, MFC175-c, and MFC225-c. The plastic bending moment of the single column cross section $\left(M_{p}\right)$ was approximately $30 \mathrm{kN} \cdot \mathrm{m}$. Therefore, assuming non-composite action between the columns, The plastic bending moment of four columns $\left(M_{o}\right)$ is equal to 120 $\mathrm{kN} \cdot \mathrm{m}$. The experimental result showed that bending strength increased with increasing embedded depth. The ratio of bending strength obtained from the test to the theoretical plastic bending moment in the cross section $\left(M_{u, \text { test }} / M_{o}\right)$ was 0.68 for MFC125-c, 0.97 for MFC175-c, and 1.23 for MFC225-c, respectively. Note that only MFC225-c had larger bending moment capacity than theoretical plastic bending moment. All test specimens had no significant strength degradation up to $4 \%$ drift ratio. 


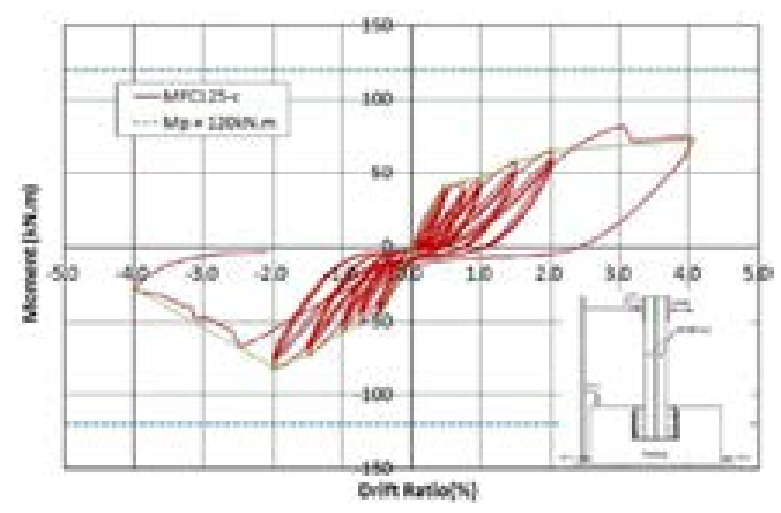

(a) MFC125-c

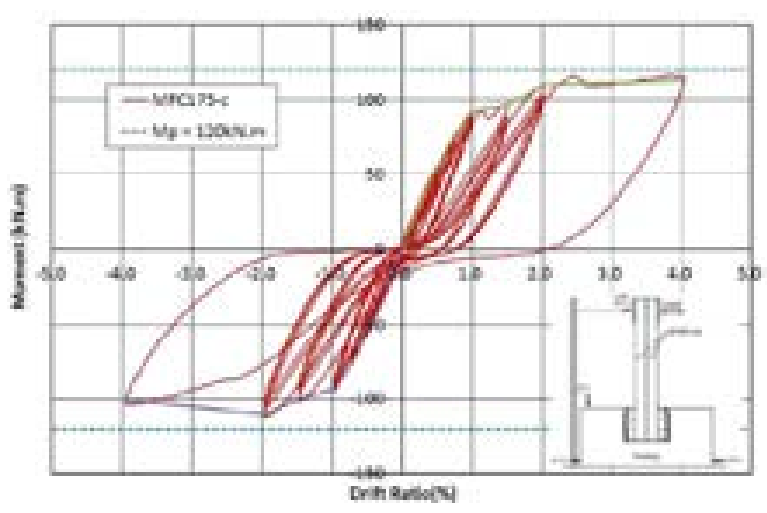

(b) MFC175-c

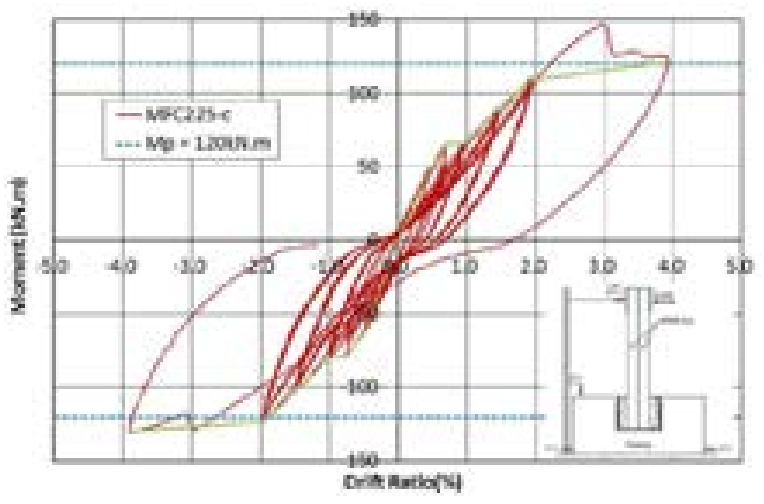

(c) MFC225-c

Figure 5 Relationship between applied moment and drift ratio for MFC125-c, MFC175-c, and MFC225-c.
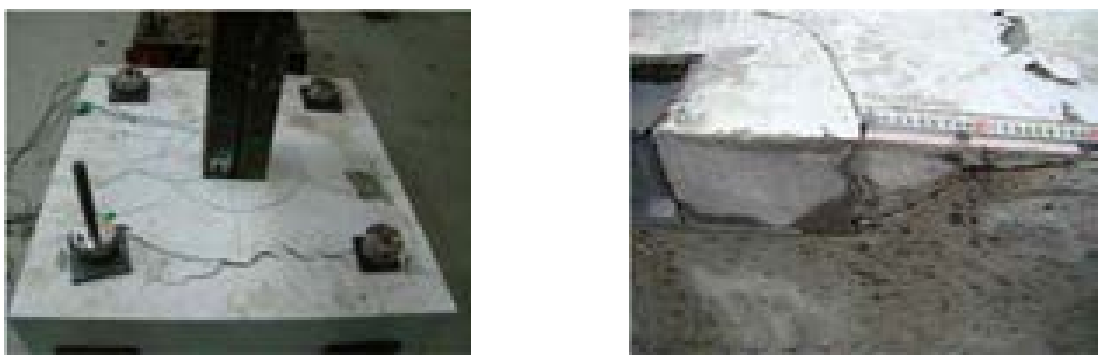

(a) MFC125-c

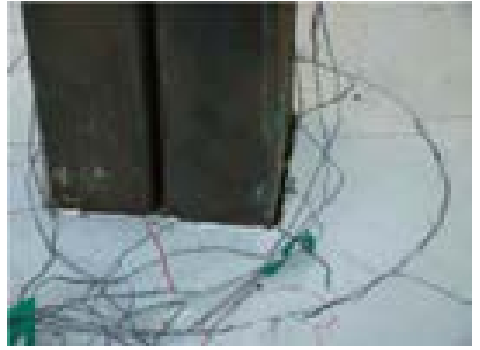

(b) MFC175-c

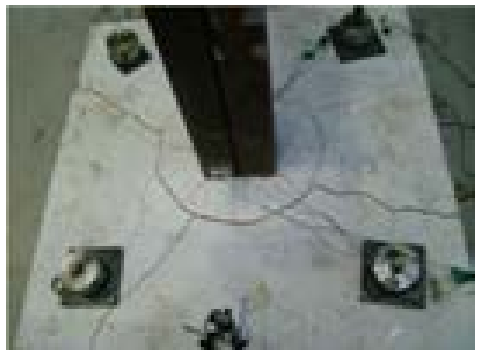

(c) MFC225-c

Figure 6 Failure shape of the connection. 
Figs. 6(a)-(c) show failure shapes of MFC125-c, MFC175-c, and MFC225-c, respectively. For MFC125-c specimen, where the embedment depth is the smallest among the test specimens, the pull-out failure occurred. Then, the foundation was cut and crack pattern was recorded as shown in Fig. 6(a). Form the crack pattern, it can be seen that cracks were developed with approximately $45^{\circ}$ angle, and initial crack was developed at the end of the base plate in tension side. Finally, the crack with $45^{\circ}$ angle reaches to the top surface of the foundation, and the pull-out failure occurred.

On the other hand, for MFC175-c and MFC225-c specimens, the cracks in the foundation are not considerable comparing with MFC125-c specimen and they showed the larger bending moment capacity than MFC125-c specimen.

Especially, the flexural strength of the MFC225-c specimens was $23 \%$ larger than theoretical plastic moment. Moreover, it can be seen that MFC225-c specimens shows the full plastic bending behavior, while shear failure of the foundation was observed in MFC125-c specimen. Thus, it can be concluded that embedment depth must be sufficient to provide the ductile flexural failure of the connection.

\subsubsection{Effect of the end plate shape and stud bolts}

Figs. 7(a) and (b) show a moment-drift ratio relationship of MFC175-cs and MFC175-d. $M_{u, \text { test }} / M_{o}$ was 0.99 for MFC175-cs and 0.93 for MFC175-d, respectively. Both specimens showed slightly lower flexural strength than to theoretical plastic bending moment.

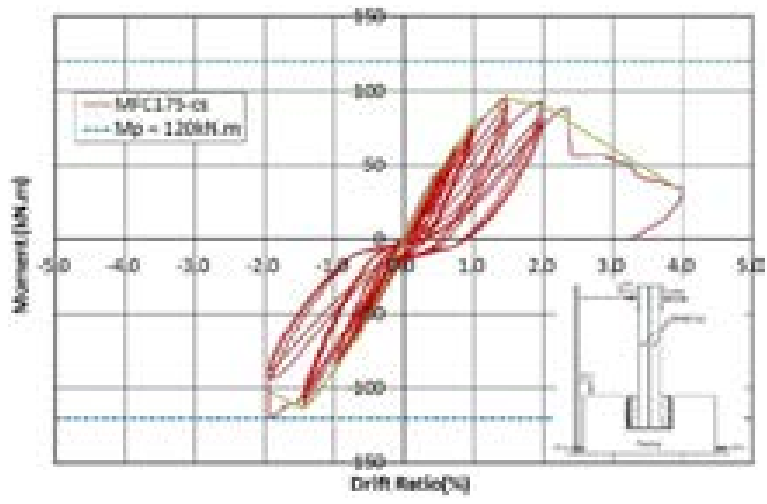

(a) MFC175-cs

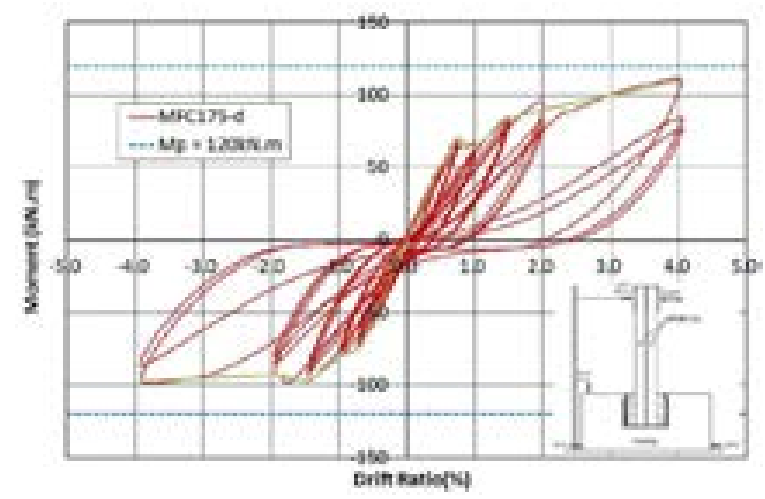

(b) MFC175-d

Figure 7 Relationship between applied moment and drift ratio for MFC175-cs, and MFC175-d.

Comparing with the test results of MFC175-c shown in Fig. 5(b), it can be seen that applied bending moment was rapidly reduced after $2.5 \%$ drift ratio for MFC175-cs specimen. It reveals that more brittle behavior occurs when the shear studs are installed in the embedded region. It seems that this is because the separation between the embedded steel column and foundation is restrained by the shear stud. Further, pull-out failure was observed in the foundation as shown in Fig. 8(a). Even if the ductility of the connection was reduced by introducing the shear studs in embedded region, the reduction in flexural strength of the connection was negligible. The shear stud may need for the proposed connection to adjust or level the column for grouting process, and more investigation of the effect of the shear stud in embedded region is required. 


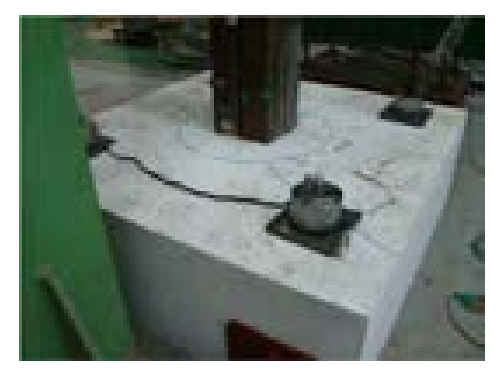

(a) MFC175-cs

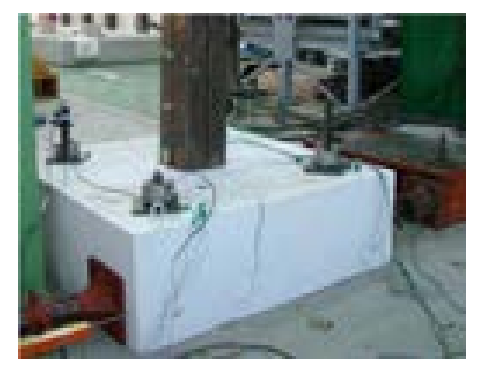

(b) MFC175-d

Figure 8 Failure shape of the foundation connection.

The effect of shape of the base plate can be evaluated by comparing the results of MFC175-c and MFC175-d specimen. From the comparison, it can be found that the flexural strength of the connection was not significantly affected by the shape of the base plate (i.e. circular or diamond shape of the base plate). However, there is little difference in applied moment-drift ratio relationship as shown in Figs. 5(b) and 7(b). Also, the failure mode was different. For the specimen with circular base plate, flexural failure of the column was dominant, while pull-out failure took place for the specimen with diamond shape base plate. In the case of circular shape of base plate, larger area of the base plate resist against pull-out failure than specimen with diamond shape base plate. Thus, stresses can be more uniformly distributed for the circular shape base plate and circular base plate may be more efficient for the proposed connection.

\section{Design equation for the proposed connection}

The failure mechanism of the proposed connection was experimentally investigated in previous sections. It was found that the pull-out failure occurs when the embedment depth is not sufficient and shear crack in the foundation is developed. On the other hand, if the embedment depth is enough, the full flexural strength of the column can be developed, and yielding of the column governs the connection behavior. Based on these observations, the simple design equation to determine the proper embedment depth was suggested in this section.
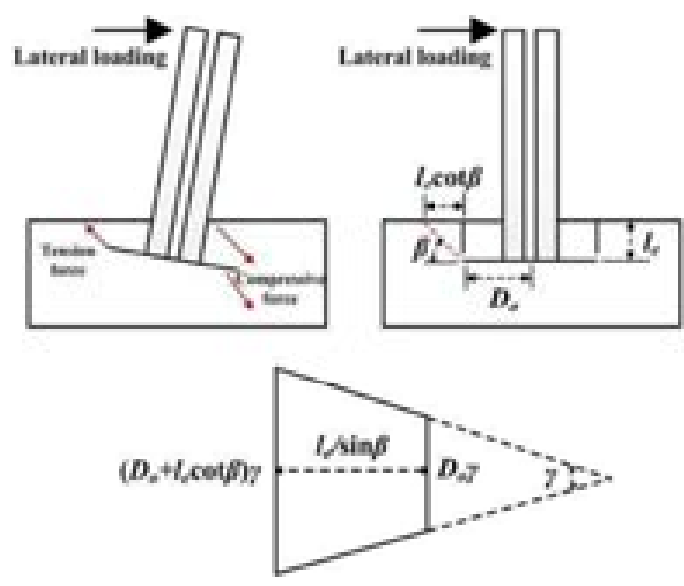

Figure 9 Pull-out failure mechanism of the proposed connection. 
Fig. 9 shows the pullout failure resistance mechanism of the proposed connection. When the lateral force is applied to the column, as shown in Fig. 9, the compressive force is developed in the right side of the foundation, while the tensile force is developed in the left side of the foundation. As a result, up-lift deformation occurs in the left side of the foundation, and shear crack can be formed.

In this study, the left side of the foundation was assumed as a vulnerable part, since concrete has small shear stress resistance than compression. Also, It is assumed that the angle of the shear crack $(\beta)$ is equal to $45^{\circ}$ based on the test results (Refer, Fig. 6(a)), and the shear crack initiates at the end of the corrugated pipe that is installed to provide the recess in the foundation. In Figure 9, $D_{o}$ represents the radius of the corrugated pipe.

From Fig. 9, the area which resists against the shear stress can be obtained as

$$
A_{\text {con }}=\gamma\left[D_{0}+\left(\frac{l_{e}}{2}\right) \cot \beta\right]\left(l_{e} \dot{s i n}\right)
$$

It is noted that the area which resists against shear stress has cone shape.

In general, concrete shear strength $\tau_{c}$ is proportional to $\sqrt{ } f_{c k}$ where $f_{c k}$ is the compressive strength of the concrete. Using this relationship, a tensile resistance force of the foundation can be calculated as

$$
T_{f}=\alpha \sqrt{f_{c k}} \gamma\left[D_{0}+\left(\frac{l_{e}}{2}\right) \cot \beta\right] l_{e}
$$

In Eq. (2), $\alpha$ is a shear strength coefficient, which can be obtained from regression analysis of the test results. In this study, it is assumed that only half of the foundation resist against the tension. Thus, $\gamma$ in Eq. (2) can be assumed as $\pi\left(180^{\circ}\right)$. Substituting $\beta=(\pi / 4)$ and $\gamma=\pi$ in Eq. (2). $T_{f}$ can be expressed as

$$
T_{f}=\alpha \sqrt{f_{c k}} \pi\left[D_{0}+\left(\frac{l_{e}}{2}\right)\right] l_{e}
$$

From the equilibrium condition, $T_{f}$ must be the same with up-lift force of the base plate $\left(T_{e}\right)$. In this study, $\gamma$ was assumed as $\pi$. The stress condition of the column when the columns are fully yielded is shown in Fig. 10. Thus, $T_{e}$ when the columns are fully yielded can be obtained by summing the tensile stress developed in the half of the base plate and it is given by

$$
T_{e}=4\left(b_{1} t-t^{2}\right) f_{y}
$$

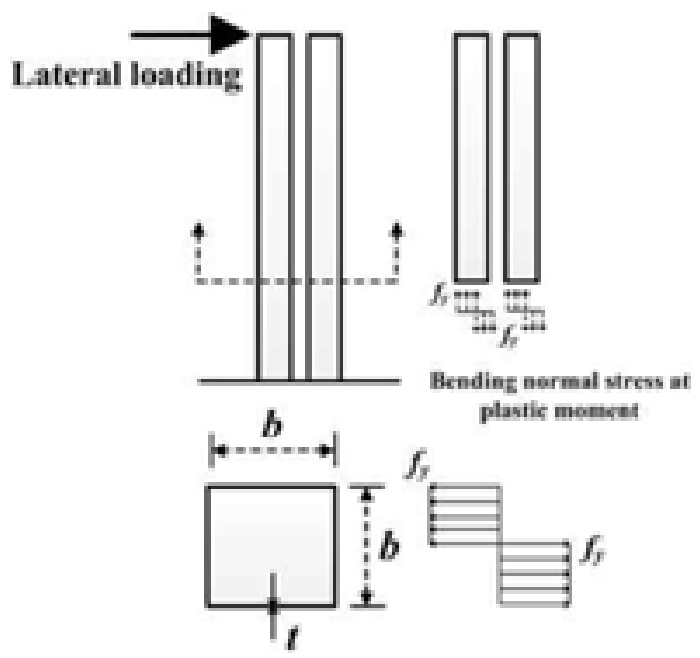

Figure 10 Distribution of bending stress of each column at the plastic moment. 
Finally, using Eq. (3) and (4), following equation is obtained:

$$
4\left(b_{1} t-t^{2}\right) f_{y}=\alpha \sqrt{f_{c k}} \pi\left[D_{0}+l_{e}+\left(l_{e} \cdot \frac{l_{e}}{2}\right)\right]
$$

Equation (5) represents the equilibrium condition when the column is fully yielded. If the shear strength coefficient $(\alpha)$ is defined, the embedment depth when columns are fully yielded (i.e. the minimum embedment depth that is required for full plastic moment of the columns) can be obtained by solving Eq. (5).

The shear strength of concretes has a large variability due to the concrete characteristic. Thus, $\alpha$ in Eq. (5) was experimentally evaluated in this study. From the test results in Section 2, $\alpha$ for each test specimens were evaluated based on Eq. (5). Then, calculated $\alpha$ was plotted with $M_{u, t e s t} / M_{p}$, as shown in Fig. 11. It can be seen that the $M_{u, \text { test }} / M_{p}$ decreasing with increasing $\alpha$, and $M_{u, t e s t} / M_{p}$ is larger than 1 when $\alpha$ is smaller than 0.47 . Finally, $\alpha$ in Eq. (5) was adopted as 0.47 . Thus, the minimum embedment depth which can develop the full plastic moments of the columns can be evaluated by Eq. (5) with $\alpha=0.47$. However, it should be noted that $\alpha$ $=0.47$ was obtained from very limited test data. Thus, more test results and analysis results should be implemented for the determination of $\alpha$ value.

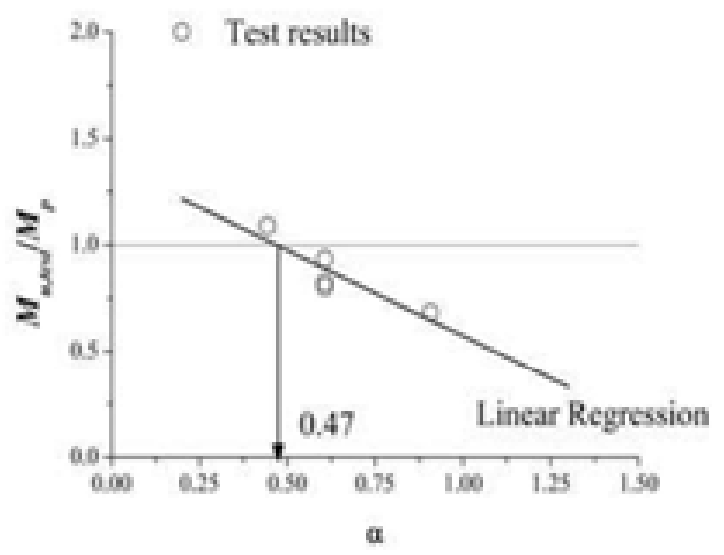

Figure 11 Determination of $\alpha$ based on Eq. (5).

In the ACI design code, the shear strength of concretes was specified as $0.33 \sqrt{ } f_{c k}$, which is $42 \%$ smaller than that proposed in this study $\left(0.47 \sqrt{ } f_{c k}\right)$. Thus, for conservative estimation of the minimum embedment depth, $\alpha$ $=0.33$ may be used.

\section{Conclusion}

In this study, failure mechanism of embedded column of the modular unit structure-to-foundation was investigated through a series of experimental study. The effect of embedment depth, shear stud in the embedded region, and the shape of the base plate were evaluated. Then, the simple deign equation for the proposed connection was suggested based on the test results. Following conclusions are obtained in this study.

1) The pull-out failure was observed when the embedment depth is not sufficient, such as MFC125-c specimen. For such specimen, concrete foundation was failed by shear crack before developing the full plastic bending moments of the columns. 
2) When the embedment depth is large enough, the full plastic bending moments of column can be obtained (MFC225-c specimen). The damage in the foundation is significantly reduced by comparing with test specimen with short embedment depth (MFC125-c specimen).

3) Shear studs in embedded region had negligible effect on the flexural strength. However, the flexural ductility was deceased, since shear studs restrains the separation of the interface between the column and concrete foundation.

4) The effect of base plate shape (Circular or diamond shape) has a little effect on the flexural behavior. However, circular base plate was more efficient, since it distribute the stress more uniformly.

5) Based on the test results, the simple design equation to determine the minimum embedment depth was proposed as Eq. (5) with $\alpha=0.47$. 0.33 for $\alpha$ specified in ACI design code also can be used for conservative estimation of the embedment depth.

\section{Acknowledgment}

This study is supported by the main project (Development of Structural Safety Technology for One day Housing) of the Korea Institute of Civil Engineering and Building Technology as well as the Housing Environment Research Project (Task No. 14RERP-B082884-01 \& 13-AUPP-C068788-03) of the Ministry of Land, Infrastructure and Transport in Korea.

\section{References}

Annan CD, Youssef MA, Naggar MH., Experimental evaluation of the seismic performance of modular steel-braced frames, Eng Struct, V.31, 2009.

Lawson RM, Ogden RG. Bergin R., Application of Modular Construction in High-Rise Buildings, J Archit Eng ASCE, V.18. No.2, 2012.

Lawson RM, Richards J., Modular design for highrise buildings, Proc Inst Civ Eng Struct Build, V.163, 2010.

Lehman DE, Roeder CW., Foundation connections for circular concrete-filled tubes, J Construct Steel Res, V.78, 2012.

Moon J, Lehman DE, Roeder CW, Lee H-E., Evaluation of embedded concrete-filled tube (CFT) column-to-foundation connections. Eng Struct, V.56, 2013.

Baltay P, Gjelsvik A. Coefficient of friction for steel on concrete at high normal stress, J Mater Civ Eng ASCE, V.2, No.1, 1990.

Moon J, Lehman DE, Roeder CW, Lee H-E, Analytical modeling of bending of circular concrete-filled steel tubes, Eng Struct, V.42, 2012.

Saenz LP., Discussion of 'Equation for the stress-strain curve of concrete' by P. Desayi, and S. Krishnan., ACI J, V.61, 1964.

American Concrete Institute (ACI), Building code requirements for structural concrete, ACI 318-11, Farmington Hills, Michigan, 2011.

Hsu TTC, Mo Y-L., Unified theory of concrete structures, 2nd edition, John Wiley \& Sons, Inc., 2010.

Lee J, Fenves GL., Plastic-damage model for cyclic loading of concrete structures, Journal of Engineering Mechanics ASCE, V.124, No.8, 1998. 